\title{
System-Level Modelling and Validation of a Strain Energy Harvesting System by Directelly Coupling Finite Element and Electrical Circuits
}

\author{
Qiang Li, Yang Kuang, Meiling Zhu \\ College of Engineering, Mathematics and Physical Science \\ University of Exeter \\ Exeter, UK \\ q.li@exeter.ac.uk, k.yang@exeter.ac.uk, m.zhu@exeter.ac.uk
}

\begin{abstract}
There is a lack of system-level finite element (FE) model which can directly predict the performance of a piezoelectric energy harvester connected with interface circuits and electric load. This work developed a system-level model of piezoelectric strain energy harvesting system by directly coupling the finite element and electrical circuits. The strain energy harvester (SEH) is a macro fibber composite adhesively bonded to a composite beam. Simulations were performed with the SEH connected with three circuits individually (i) a load resistor, (ii) a rectifier terminated with a load resistor and (iii) a rectifier terminated with a smoothing capacitor and a load resistor. Experimental tests were carried out to validate the simulation results. Good agreements were observed between the simulated and measured results. The developed model is able to predict the performance of the energy harvesting system when different circuit was connected. The validated system-level model can be used for the design and optimization of piezoelectric energy harvesting system by investigating the interactions between energy harvester and electrical circuits.
\end{abstract}

Keywords-system-level modelling; piezoelectric energy harvesting; Finite Element Analysis; macro fibber composite

\section{INTRODUCTION}

Energy harvesting powered wireless sensors for structural health monitoring (SHM) have received growing attentions over the past decade[1], [2]. This has been motivated by the desire to get rid of maintenance such as battery recharging or replacement and to increase the life time of the sensors. For structures exposed to cyclic strains during operation, the most abundant energy source is strain energy, which can be scavenged by using a piezoelectric energy harvester (PEH). A piezoelectric element can be bonded to the target structure. When the host structure is cyclically strained during operation, the piezoelectric element is also stressed and thus generates electric energy by direct piezoelectric effect.

A typical piezoelectric energy harvesting system (PEHS) usually comprises a $\mathrm{PEH}$ to generate energy, an interface circuit (IC) to regulate the generated energy and a working unit or an equivalent electric load representing the working unit. While the PEH generates electric energy by using direct piezoelectric effect, the dynamic behaviours of the succeeding circuit (the IC and the electric load) affects the PEH by inverse piezoelectric effect [3]. Moreover, the output of the PEH can also vary the performance of the succeeding circuit. Therefore, the overall efficiency of a PEHS is not only dependent on the energy harvester performance but also on the succeeding circuit design. While experimentally studying the effects of these factors is costly and time-consuming, there is a need to develop a system-level model to couple both the PEH and the succeeding circuit to perform coupled analysis. However, due to the difficulty in direct combination of finite element (FE) analysis and nonlinear interface circuits, the system-level models reported in the literature still simulate the PEH and the succeeding circuit separately and cannot reflect the coupling between them[3]-[5]

This work reports the development and experimental validation of a system-level model of a piezoelectric strain energy harvesting system, which directly couples a strain energy harvester (SEH) and the succeeding circuit. The developed model is able to perform system-level analysis and thus provides a powerful tool for piezoelectric energy harvesting system design and optimisation.

\section{STRAIN ENERGY HARVESTING SYSTEM}

The strain energy harvester studied in this paper is shown in Fig. 1. A macro fiber composite (MFC) is adhesively bonded by $20 \mu \mathrm{m}$-thick epoxy to a composite beam, which is an embodiment of an engineering structure subject to cyclic strains. The MFC comprises PZT fibbers buried in epoxy resin. When the composite beam is strained, a stress is developed in the MFC, which generates electric energy by direct piezoelectric effect. Fig. 2 shows a schematic of the piezoelectric strain energy harvesting system. The AC voltage generated by the SEH is converted to DC by a full-bridge rectifier, the output of which is smoothed by a $20 \mu \mathrm{F}$ capacitor and then terminated with a load resistor.

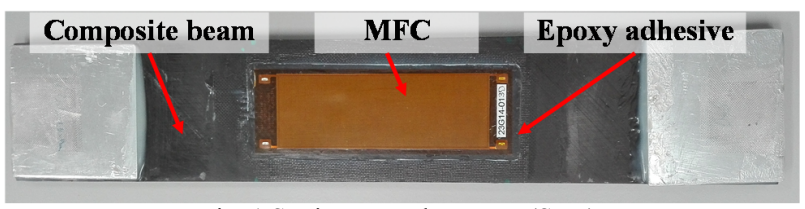

Fig. 1 Strain energy harvester (SEH) 


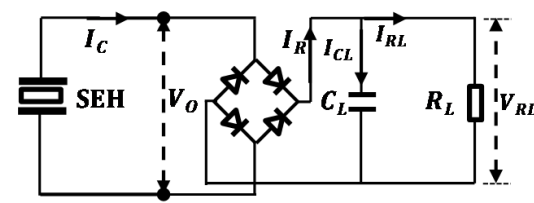

Fig. 2 Diagram of a strain energy harvesting system

\section{SYSTEM-LEVEL MODELLING AND EXPERIEMTNAL VALIDATION METHODS}

\section{A. Directly coupled system-level model}

The directly coupled system-level model was developed using commercial package COMSOL. A schematic of the model is shown in Fig. 3. The MFC was modelled as a layer of solid homogeneous piezoelectric material rather than PZT fibres buried in epoxy resin to simplify the physical model. The piezoelectric layer was coupled to the composite beam through an epoxy layer. The dimensions of the piezoelectric material layer, epoxy layer and the beam were kept the same as the SEH shown in Fig. 1. The electric potential of the top and bottom surfaces of the piezoelectric layer were coupled individually to serve as two electrodes, which were connected to the succeeding circuits. Three succeeding circuits were used in simulation to demonstrate the ability of the model to predict the performance of the SEH with different circuits. The three circuits are illustrated in Fig. 3: (i) a load resistor, (ii) a rectifier terminated with a load resistor and (iii) a rectifier terminated with a smoothing capacitor $(22 \mu \mathrm{F})$ and load resistor.

One end of the beam was fixed by applying displacement constraints while the other end was applied with a harmonic force along the length direction to generate the expected strain level (100 $600 \mu \varepsilon$ peak-to-peak). To avoid the compression of the beam, a static tensile force was applied. Therefore, the total load force $F_{\text {load }}$ applied takes the force of

$$
F_{\text {Load }}=F_{a}(1+\sin (1.5 \pi+2 \pi f t))
$$

where $F_{a}$ is the force amplitude and $f$ is the frequency. The harmonic force has an initial phase of $1.5 \pi$. This makes sure that the load force starts from zero.

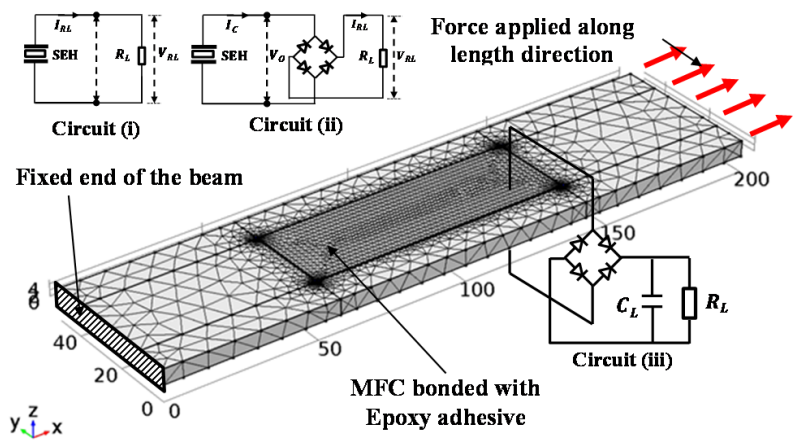

Fig. 1 Modelling of piezoelectric strain energy harvesting system

\section{B. Experimental validation methods}

To validate the simulation results, the SEH shown in Fig. 1 was installed on a material testing machine (INSTRON 1000), as shown in Fig. 4. The material testing machine was programed to apply a harmonic tensile force on the beam to generate the expected strain level, which was measured by an extensometer. The output of the SEH was connected with one of the aforementioned three succeeding circuits each time and the energy generation was monitored by using a data acquisition card.

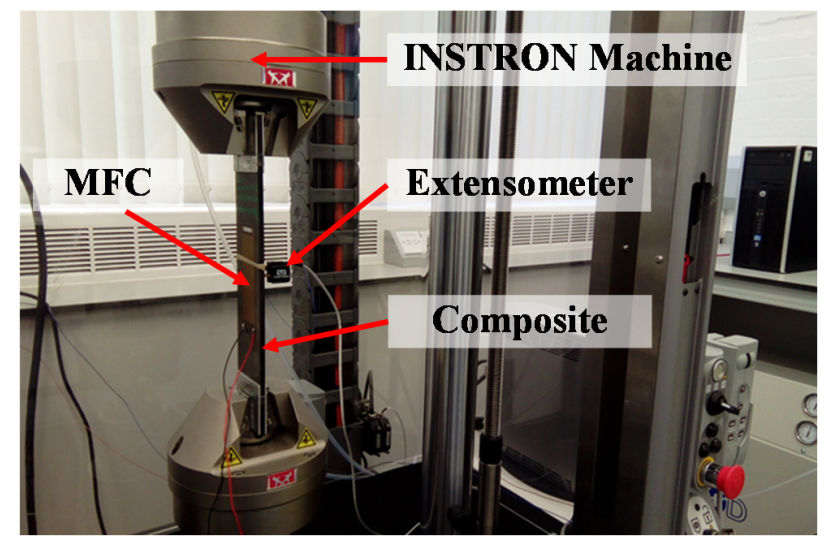

Fig. 2 Experimental setup for model validation

\section{RESULTS AND DISCUSSION}

\section{A. Model validation}

Fig. 5 compares the simulated and measured outputs of the SEH connected with circuit (i) when a strain of $600 \mu \varepsilon$ at different frequencies were applied. The simulation results agree with the experiment. At each strain frequency, the voltage first increases with the load resistor and then gradually stabilises at the open-circuit voltage. Maximum power output is observed at the optimal load resistance, which matches the internal impedance of the SEH. The optimal load resistance varies with strain frequency because the internal impedance of the piezoelectric material is dependent on the frequency.

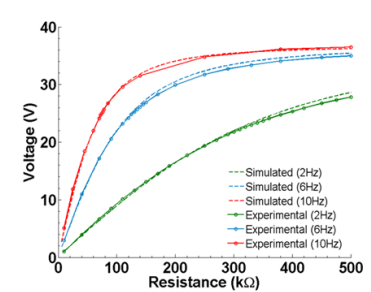

(a)

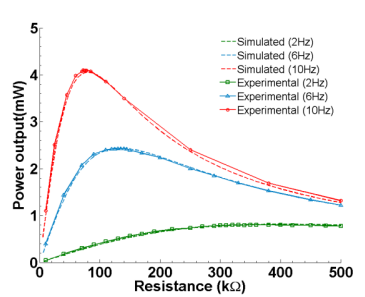

(b)
Fig. 3 Comparison of the simulated and measured results when the SHE is connected with circuit (ii) (a) voltage vs. load resistance (b) power vs load resistance

Fig. 6 shows the simulated and measured power outputs of the SEH connected with circuit (ii) as the SEH was excited at different strain frequencies. As before, good agreement is observed between the simulation and experiment. In Fig. 6 (a), the power output demonstrates the similar dependence on the load resistance and the strain frequency as Fig. 5 (b). Fig. 6 (b) shows the dependence of the power output on the strain frequency and amplitude when the corresponding optimal load resistor was connected. Both simulation and experiment results 
show that the power output increases approximately linearly with the strain frequency.

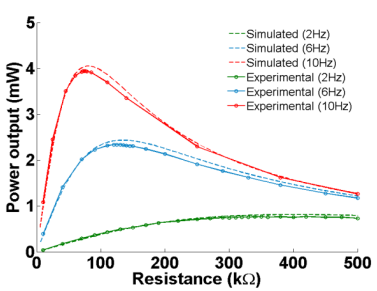

(a)

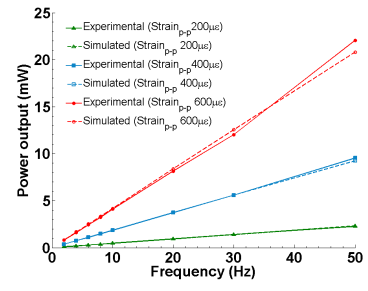

(b)
Fig. 4 Comparison between the simulated and measured power output when the SEH is connected with circuit (ii) (a) power output vs. load resistance (b) power output vs strain frequency

Fig. 7 presents the simulated voltages and currents when the SEH was connected to the succeeding circuit (iii). The SEH was actuated harmonically with a strain of $600 \mu \varepsilon$ at $10 \mathrm{~Hz}$. As a result, a harmonic voltage was generated by the piezoelectric material. The voltage across the load resistor increases quickly with time and then stabilises, as expected when a smoothing capacitor is used. The voltage across the load resistor is slightly lower than the voltage amplitude generated by the MFC. This is caused by the energy loss on the rectifier.

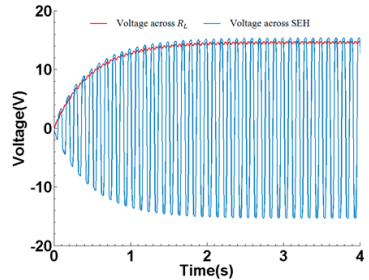

(a)

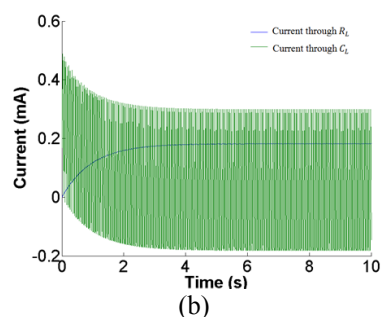

(b)
Fig. 7 Simulated voltage (a) current (b) when the SHE was connected with the succeeding circuit (iii)

When the SEH was connected to the succeeding circuit (iii), the simulated and measured voltage and energy in the load resistor are compared in Fig. 8 (a) with a detained plot in (b). The simulation results coincide with the experiment.

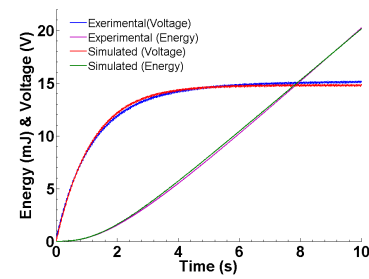

(a)

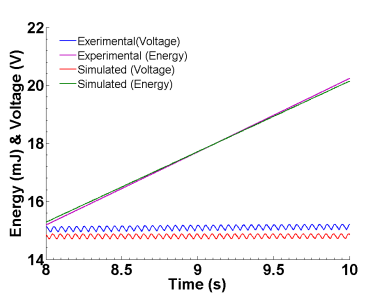

(b)
Fig. 5 Comparison of voltage and harvested energy

\section{B. Further Simulation Results}

The validated system-level model can be used to perform design studies of the strain energy harvesting system. As a case study, simulations were performed to investigate the effect of the bonding layer (epoxy) thickness on the power output of the
SEH when it was actuated at a strain of $600 \mu \varepsilon$ at $10 \mathrm{~Hz}$ and connected with the succeeding circuit (i). As the epoxy thickness increases, the power output of the SEH decreases, as shown in Fig. 9. This is because the strain energy from the composite beam is transferred to the piezoelectric material by the epoxy layer. As the epoxy thickness increases, more strain energy is dissipated in the epoxy with a reduced strain energy transferred to the piezoelectric material.

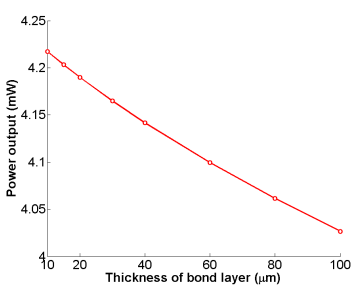

Fig. 9 Effects of the epoxy thickness on the power output

\section{CONCLUTIONS}

A system level model for a piezoelectric strain energy harvesting has been developed and experimentally validated. The model directly coupled the FE model of the SEH with different circuits. It is able to simulate the electric energy output of the SEH as well as the energy loss and distribution in the system, when the SEH was actuated with different strain amplitudes and frequencies. This model can be used to study the overall efficiency of a piezoelectric energy harvesting and provide guidelines for system design and optimisation.

\section{REFERENCES}

M. Q. Le, J.-F. Capsal, M. Lallart, Y. Hebrard, A. Van Der Ham, N. Reffe, L. Geynet, and P.-J. Cottinet, "Review on energy harvesting for structural health monitoring in aeronautical applications," Prog. Aerosp. Sci., vol. 79, pp. 1-11, 2015.

[2] A. Giuliano, V. Marsic, and M. Zhu, "Implementation and testing of an elastic strain powered wireless sensing system for energyautonomous applications," Proc. - 2012 IEEE Int. Conf. Green Comput. Commun. GreenCom 2012, Conf. Internet Things, iThings 2012 Conf. Cyber, Phys. Soc. Comput. CPSCom 2012, pp. 681-684, 2012.

[3] C. Cheng, Z. Chen, H. Shi, Z. Liu, and Y. Xiong, "System-Level Coupled Modeling of Piezoelectric Vibration Energy Harvesting Systems by Joint Finite Element and Circuit Analysis," 2016.

[4] P. H. Wu and Y. C. Shu, "Finite element modeling of electrically rectified piezoelectric energy harvesters," Smart Mater. Struct., vol. 9057, pp. 1-15, 2015.

[5] B. Bayik, A. Aghakhani, I. Basdogan, and A. Erturk, "Equivalent circuit modeling of a piezo-patch energy harvester on a thin plate with AC-DC conversion," Smart Mater. Struct., vol. 25, no. 5, p. $055015,2016$. 